\title{
Case Report \\ Entanglement due to Delayed Removal of a Buddy Wire
}

\author{
Marc Vorpahl, Melchior Seyfarth, and Klaus Tiroch \\ Department of Cardiology, HELIOS Klinikum Wuppertal, Witten/Herdecke University, Arrenberger Strasse 20, \\ 42117 Wuppertal, Germany \\ Correspondence should be addressed to Marc Vorpahl; marc.vorpahl@helios-kliniken.de
}

Received 6 October 2014; Accepted 17 December 2014; Published 29 December 2014

Academic Editor: Kenei Shimada

Copyright (c) 2014 Marc Vorpahl et al. This is an open access article distributed under the Creative Commons Attribution License, which permits unrestricted use, distribution, and reproduction in any medium, provided the original work is properly cited.

\begin{abstract}
A buddy wire is often used to aid in the delivery of balloons and stents when negotiating tortuous or calcified vessels. We present a planned two-stent mini-crush intervention complicated by entanglement of the buddy wire with the second stent and subsequent distortion of the stent within the guiding catheter. Based on this case, we suggest removing the buddy wire immediately after successful positioning of the first stent, because entrapment with a second stent is possible and may lead to challenging situations in a simultaneous two-stent strategy.
\end{abstract}

\section{Introduction}

Placement of a second coronary guide wire parallel to the working wire can be considered for challenging percutaneous coronary interventions (PCI). Such situations include calcified lesions, tortuous vessels, and sharp bends. This "buddy wire" stabilizes the guiding backup and position by straightening of the target vessel [1]. It is recommended to remove the "buddy wire" after stent positioning and before stent implantation to avoid entrapment of the buddy wire between the vessel and the stent. Treatment of a complex bifurcation lesion with the "mini-crush" technique using two simultaneous stents has been well described $[2,3]$; however, the timing of removing a buddy wire during stent delivery is not well established.

\section{Case Report}

An 86-year-old female presented with acute decompensated heart failure and non-ST elevation myocardial infarction. Echocardiography demonstrated severe impaired systolic left ventricular function and moderate mitral regurgitation. Cardiac catheterization revealed severe coronary artery disease with significant stenosis of the distal left main artery (LM) and the ostial/proximal left circumflex (LCx) (Figures 1(a) and $1(\mathrm{~b})$ white arrows) and intermediate stenosis of the proximal left descending artery (LAD). Based on a EuroSCORE II of 36, the Heart Team decision was in favor of a percutaneous revascularization strategy. The upfront plan was to treat the left main bifurcation with a simultaneous two-stent "minicrush" strategy. The calcified and angulated proximal LCx required a "buddy wire" (FIELDER Coronary Guide Wire, ASAHI) in addition to the working wire (ChoICE PT Extra Support Guide Wire, Boston Scientific). Another FIELDER wire was placed into LAD. Predilation of the LAD and LCx (2.0 and $2.5 \times 20 \mathrm{~mm}$, NC Trek, 20 atm., Abbott Vascular) occurred smoothly, and the first stent (Xience PRO $3.0 \times$ $28 \mathrm{~mm}$, Abbott Vascular) was successfully advanced into the LCx over the ChoICE PT working wire. Then, we advanced the second stent into the LAD (Xience PRO $3.0 \times 28 \mathrm{~mm}$, Abbott Vascular). The advancement of the LAD stent was effortless within the guiding catheter except for the last few centimeters and within the left main, where increasing friction impeded further advancement. Surprisingly, initial attempts to pull the LCx "buddy wire" failed after successful advancement of both stents most likely related to the twisted LCx buddy wire around the LAD wire/LAD stent (Figure 1(c)). Due to entanglement, forced pullback maneuvers of the buddy wire tore the LAD stent catheter tip off the shaft, blocking the mid-part of the 7F Guiding EBU 4.0 (Medtronic) (Figure 1(e)). Fortunately, we were able to deploy left main/LCx stent without significant plaque shifting into the LAD, leaving an excellent single stent result (Figure 1(d)). The guide catheter was removed, revealing 


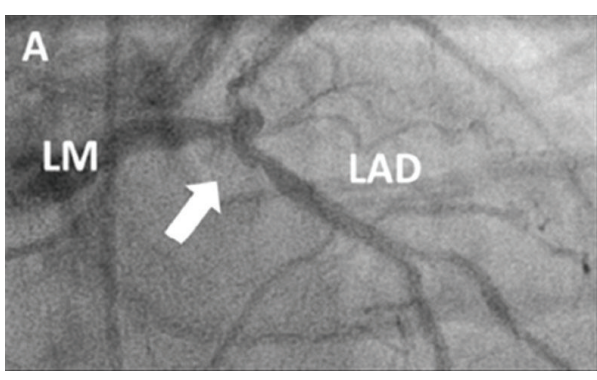

(a)

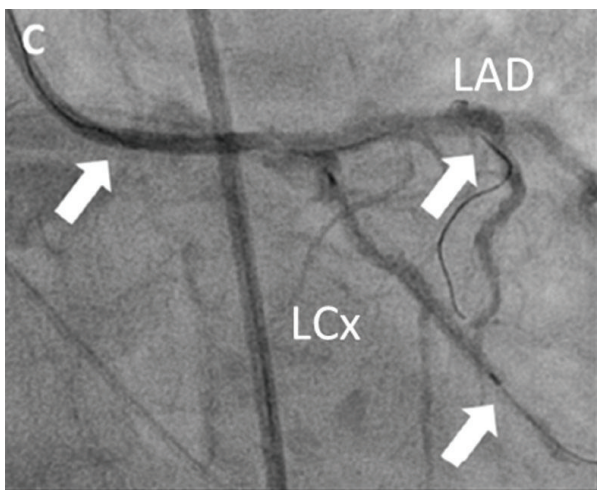

(c)

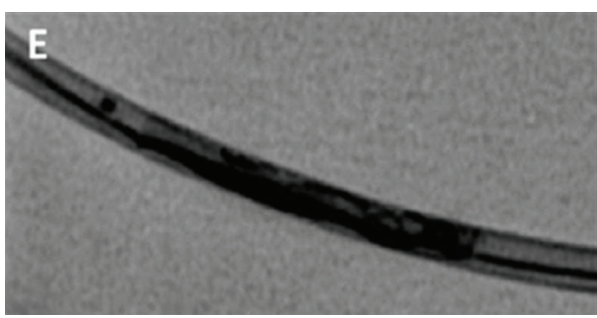

(e)

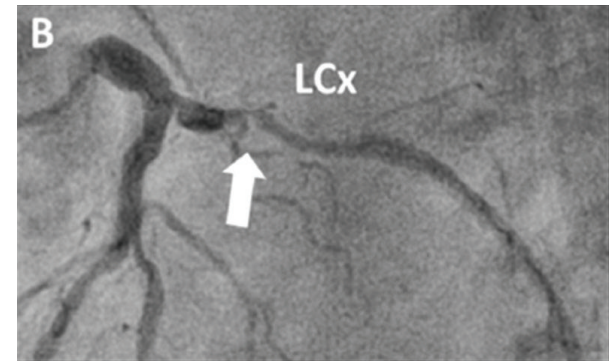

(b)

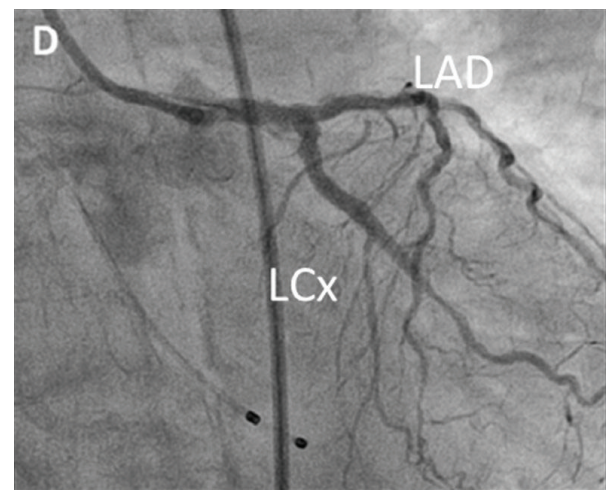

(d)

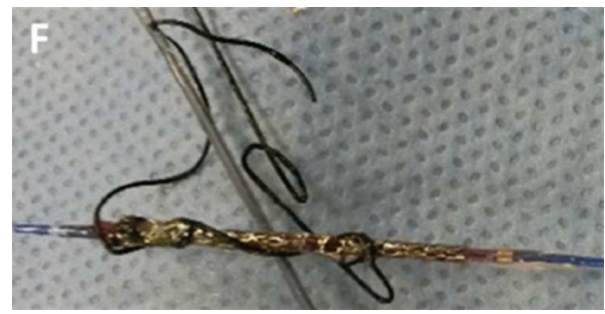

(f)

FIGURE 1: (a) and (b) Angiogram showing severe coronary artery disease with significant stenosis of the distal left main artery (LM) and the proximal left circumflex (LCx) (white arrows). (c) Mini-crush stenting was planned with one wire in the LAD and 2 wires including the buddy wire in the LCx. Advancement of the second stent into the LAD leads to entrapment and distortion of this stent by the buddy wire after successful positioning of the first stent into the LCx. (d) Provisional stenting of the LM-LCx lesion showed a good final result. (e) and (f) Attempts to pull the LCx "buddy wire" failed directly after advancement and before expansion of stents. Forced pullback of the buddy wire tore the LAD stent catheter tip off the shaft, blocking the mid-part of the 7F Guiding.

the severely entangled stent wrapped by the FIELDER "buddy wire" (Figure 1(f)).

\section{Discussion}

The "buddy wire" is a well-accepted technique for advancement of balloons and stents in calcified lesions, sharp bends, and tortuous vessels. During a complex bifurcation PCI, the first stent should be placed parallel to the buddy wire in order to gain optimal support. With a planned two-stent strategy, however, there is a certain reluctance to remove the buddy wire prematurely before final positioning of stents. In our case though, we experienced an entanglement of the buddy wire during advancement of the second stent with subsequent disruption of the stent. We therefore suggest removing the buddy wire immediately after successful positioning of the dedicated first stent, because entrapment with a second stent is possible and may lead to challenging situations in a planned simultaneous two-stent strategy.

\section{Conclusion}

In a planned simultaneous two-stent strategy, we suggest removing the buddy wire immediately after successful positioning of the first stent to avoid entanglement of the second stent with the buddy wire.

\section{Conflict of Interests}

The authors declare that there is no conflict of interests regarding the publication of this paper. 


\section{References}

[1] F. Burzotta, C. Trani, M. A. Mazzari et al., "Use of a second, 'buddy' wire during percutaneous coronary interventions: a simple solution for some challenging situations," Journal of Invasive Cardiology, vol. 17, no. 3, pp. 171-174, 2005.

[2] A. Colombo, G. Stankovic, D. Orlic et al., "Modified T-stenting technique with crushing for bifurcation lesions: Immediate results and 30-day outcome," Catheterization and Cardiovascular Interventions, vol. 60, no. 2, pp. 145-151, 2003.

[3] A. R. Galassi, A. Colombo, M. Buchbinder et al., "Long-term outcomes of bifurcation lesions after implantation of drugeluting stents with the 'mini-crush technique," Catheterization and Cardiovascular Interventions, vol. 69, no. 7, pp. 976-983, 2007. 


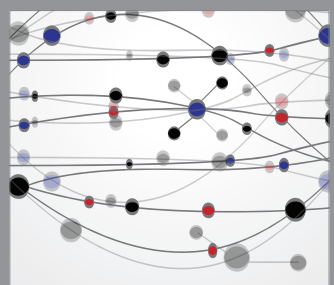

The Scientific World Journal
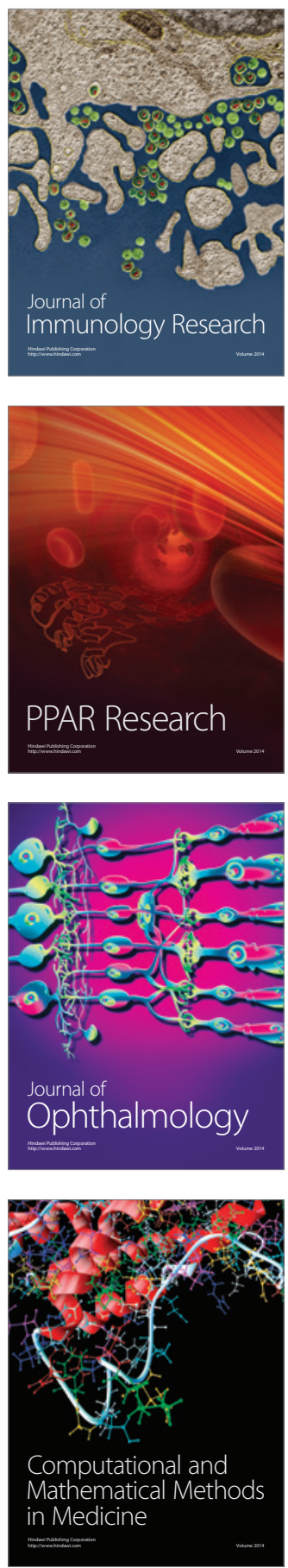

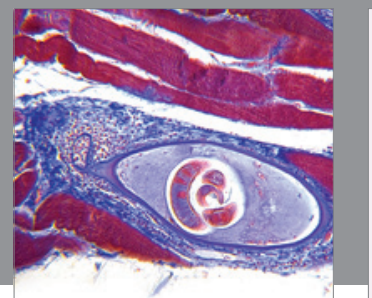

Gastroenterology

Research and Practice
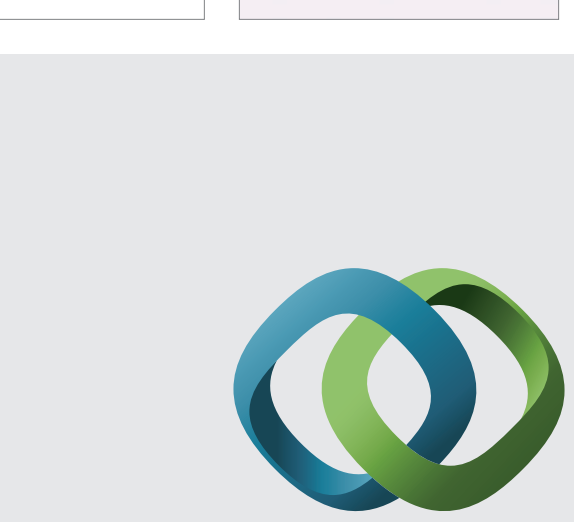

\section{Hindawi}

Submit your manuscripts at

http://www.hindawi.com
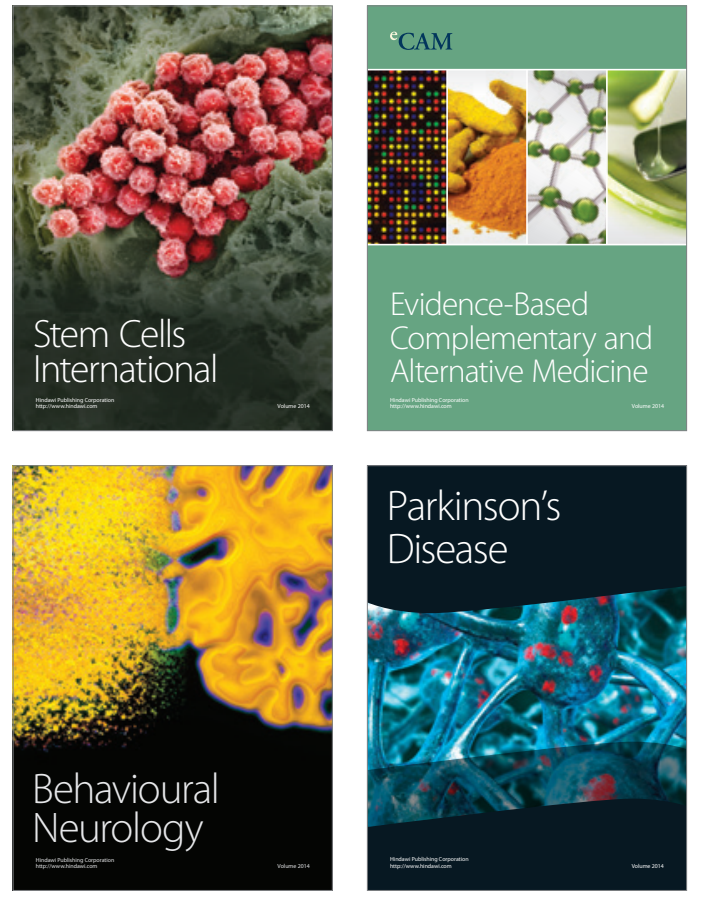
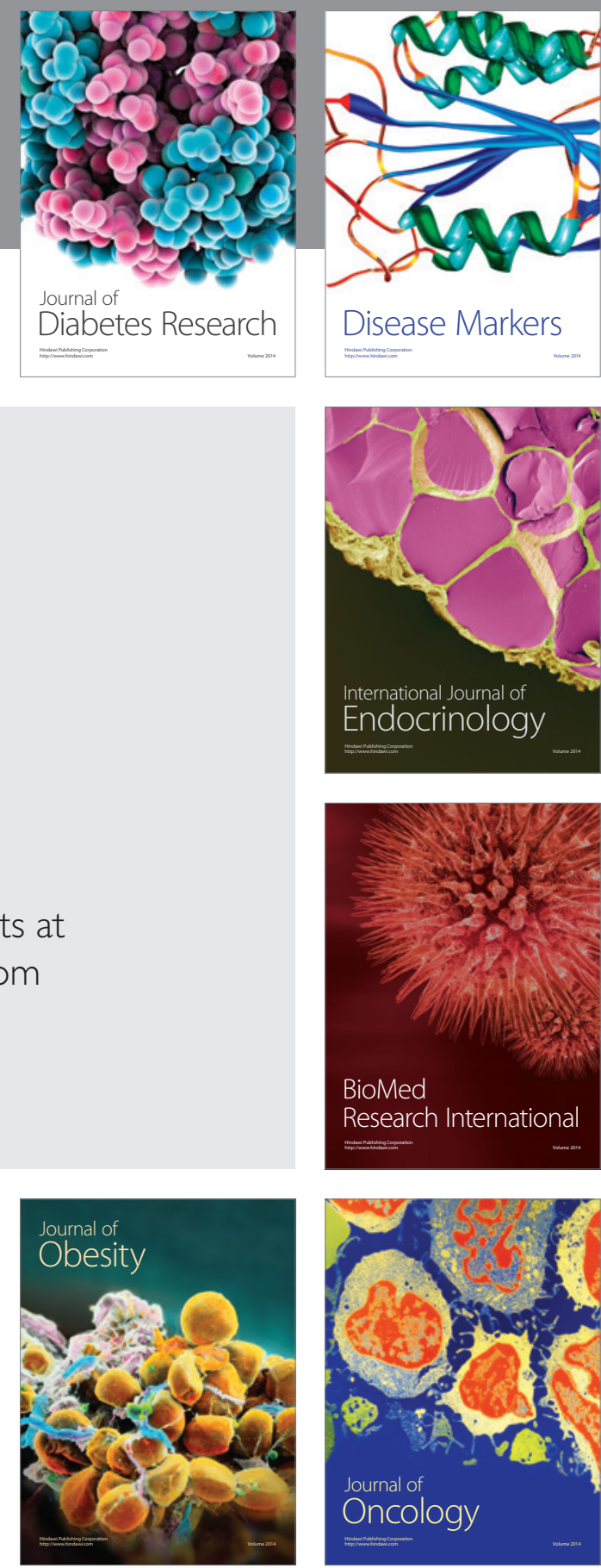

Disease Markers
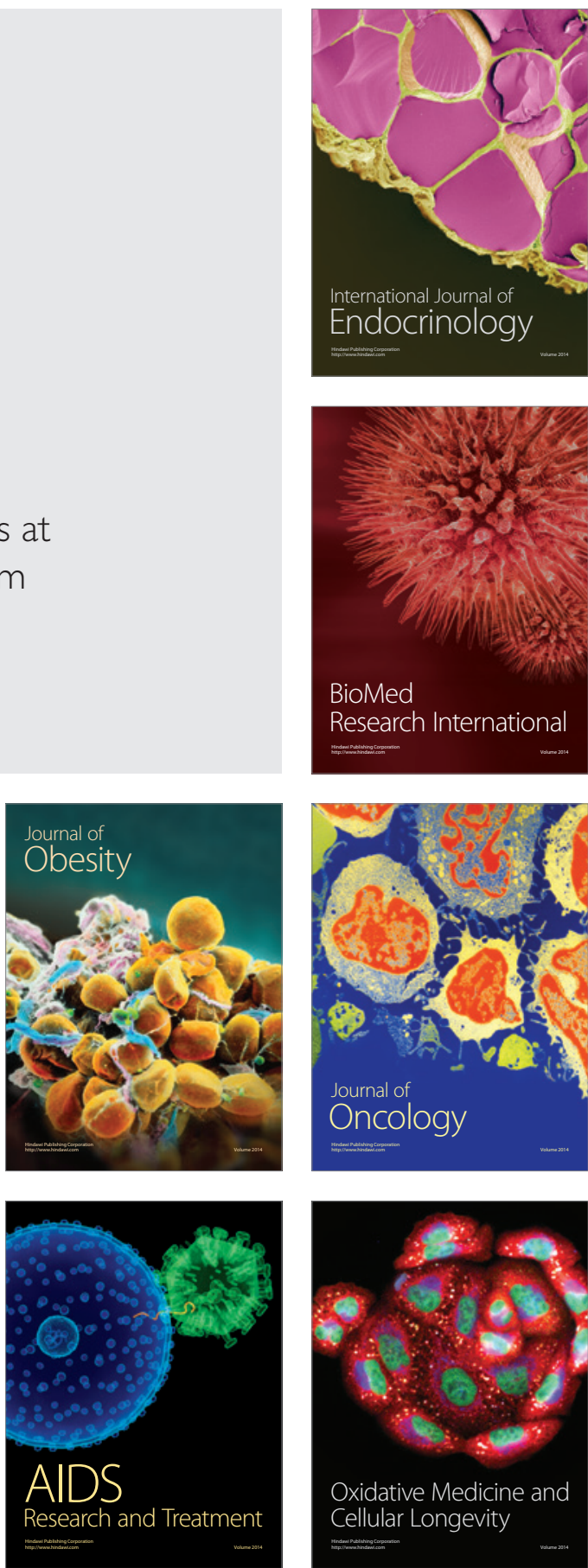